\title{
A global optimization technique for the fitting of color-magnitude diagrams
}

\author{
Hektor Monteiro, Wilton S. Dias and Thiago C. Caetano \\ Universidade Federal de Itajubá, Itajubá, MS, Brazil \\ email: hektor.monteiro@gmail.com
}

\begin{abstract}
We present a new technique to analyze color-magnitude diagrams, such as those of stellar clusters, based on the cross-entropy global-optimization procedure. The method uses theoretical isochrones available in the literature and minimizes an objective function that describes the quality of a fit to the color-color and color-magnitude diagrams. The objective function is based on the two-dimensional distances of the points in the color-magnitude and color-color diagrams to the theoretical curves and is modified by weights that take into account the stellar distance to the observed cluster center, observed magnitude uncertainties and the King profile of the cluster, among others. The parameters determined simultaneously are distance, reddening, age and metalicity. The method uses a Monte Carlo approach to obtain uncertainties on the determined parameters for the cluster. We present results for 10 well-studied open clusters and show that the results are consistent with previous studies.
\end{abstract}

Keywords. open clusters and associations: general methods: numerical, methods: statistical

\section{Introduction}

Galactic open clusters are a key class of objects used for a wide range of investigations owing to their broad age and distance span. However, main-sequence 'fitting' to determine those parameters in most studies to date has been done with subjective 'by eye' fitting. This is mainly due to the fact that the isochrones have no simple parametric form and are usually in the form of tabulated points for a set of fundamental parameters such as age and metallicity. The traditional 'fitting' method used is to first determine the reddening by adjusting the zero-age main sequence (ZAMS) to the observed color-color diagram - usually $(B-V)$ versus $(U-B)$ - of the cluster and subsequently, keeping this value fixed, adjusting the distance and age using the observed color-magnitude diagram (CMD) and tabulated isochrones. The subjectivity in the determination of the reddening is particularly problematic since it affects the subsequent determination of the distance and age of the cluster. It is also important to point out that in some cases the reddening is not determined through the use of $U$-band photometry, which can further compromise the results obtained.

In this paper, we present a new technique to fit models to open cluster photometric data using a geometric criterion to define the goodness of fit and a global optimization algorithm known as cross entropy (CE) to find the best-fitting isochrone.

\section{The cross-entropy optimization method}

The CE procedure provides a simple, adaptive way of estimating the optimal reference parameters. Basically, the CE method involves an iterative procedure where in each iteration the following is done:

1. Random generation of the initial parameter sample, respecting pre-defined criteria;

2. Selection of the best candidates based on some mathematical criterion; 

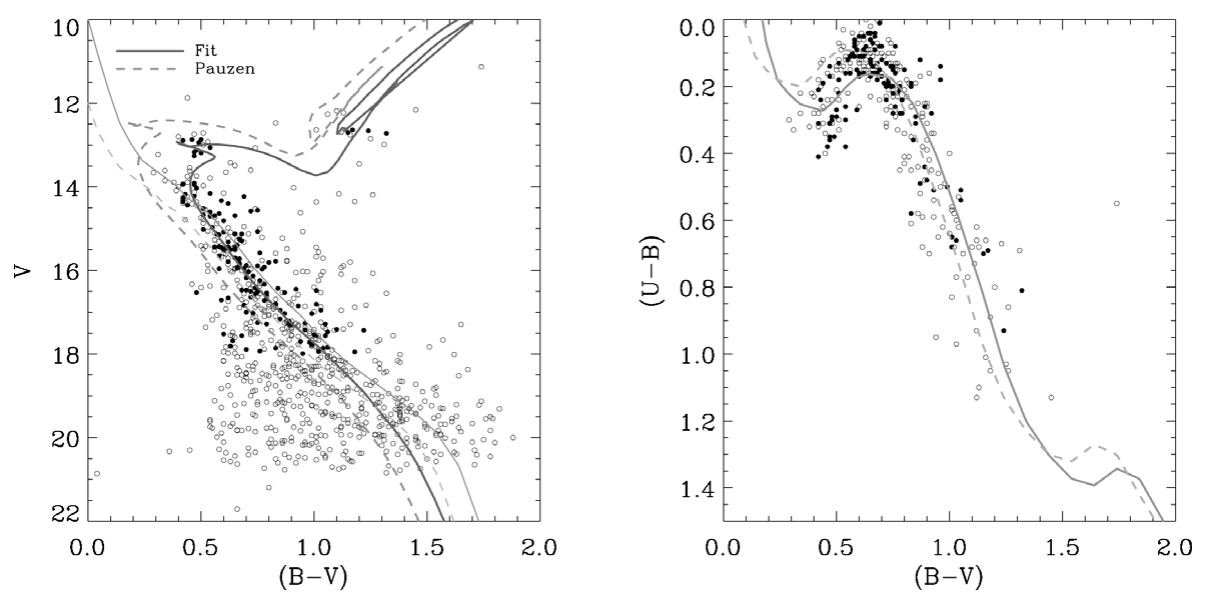

Figure 1. Example of the final fit results for NGC 2355.

3. Random generation of updated parameter samples from the previous best candidates to be evaluated in the next iteration;

4. Repeat steps $(b)$ and $(c)$ until a pre-specified stopping criterion is met.

For isochrone fitting, we require that a good solution be one that minimizes the sum of the minimum distances of the data points in the $(V, B-V)$ plane to the model. Ideally, any point would sit on the model curve and the variances as well as the minimal distances would be zero for a perfect solution. The sum of the variances is also modified by weights for each data point. Thus, our objective function is given by:

$$
S=\sum_{i} \min \left[\frac{\left(V_{i}-I_{V}\right)^{2}}{\sigma_{V_{i}}^{2}}+\frac{\left((B-V)_{i}-I_{(B-V)}\right)^{2}}{\sigma_{(B-V)_{i}}^{2}}\right] \times W_{i},
$$

where $I$ is the tabulated isochrone function, $V$ the observed $V$-band magnitude, $(B-V)$ the color index, $\sigma_{V_{i}}$ the error on the $V$ magnitude of point $i, \sigma_{(B-V)_{i}}$ the error on the $(B-V)$ color of point $i$ and $W_{i}$ is the weight of point $i$ based on its probability of belonging to the cluster.

The tabulated isochrones are defined by four parameters, i.e., age distance, extinction and metallicity. The parameter ranges are pre-defined by the user and should be representative of the problem being optimized. In our isochrone fitting we defined the parameter space as follows:

1. Age: from $\log ($ age $/ y r)=6.6$ to 10.15 ;

2. Distance: from 1 to $10000 \mathrm{pc}$;

3. $E(B-V)$ : from 0.0 to $3.0 \mathrm{mag}$.

To determine the weights of each observed star, we must first remove the contamination from stars that do not belong to the cluster. The contamination is usually from field stars that can be at different distances and have different reddening values than the cluster. Therefore, we introduce schemes to filter out contaminating stars and determine which stars are more likely to belong to the cluster in a way that can be easily reproduced given simple and clear parameters.

The weight estimation for each star is performed by comparing the characteristics of the stars in an area around the $i^{\text {th }}$ star in the CMD defined by a box with dimensions $3 \sigma_{V_{i}} \times 3 \sigma_{(B-V)_{i}}$. We then calculate the average and standard deviation of $V$ and $(B-V)$ for the stars that fall within this box and that are at a radius within the cluster region defined through the stellar density profile. We then calculate the probability of this star 
Table 1. Basic parameters for the investigated clusters newly determined here. The numbers in the final column are the WEBDA reference codes, $E(B-V)$ is the extinction, $d$ the distance to the cluster, $\log$ (Age) the logarithmic age (in years), $Z$ the adopted metallicity and $\chi_{\text {red }}^{2}$ the reduced $\chi^{2}$. The literature values are from Paunzen \& Netopil (2006).

Fit

\begin{tabular}{|c|c|c|c|c|c|c|c|c|c|c|}
\hline Clus & $\begin{array}{c}E(B-V) \\
(\mathrm{mag})\end{array}$ & $\begin{array}{l}\text { Distance } \\
(\mathrm{pc})\end{array}$ & $\begin{array}{c}\log (\text { Age }) \\
(y r)\end{array}$ & $Z$ & $\overline{\chi_{\text {red }}^{2}}$ & $\begin{array}{c}E(B-V) \\
(\mathrm{mag})\end{array}$ & $\begin{array}{l}\text { Distance } \\
(\mathrm{pc})\end{array}$ & $\begin{array}{c}\log (\text { Age }) \\
(y r)\end{array}$ & $\chi_{\mathrm{red}}^{2}$ & Ref. \\
\hline NGC 2477 & $0.26 \pm 0.01$ & $1349 \pm 20$ & $8.95 \pm 0.06$ & 0.019 & 2.9 & $0.26 \pm 0.08$ & $1227 \pm 166$ & $8.94 \pm 0.11$ & 3.3 & 152 \\
\hline NGC 7044 & $0.55 \pm 0.05$ & $3383 \pm 215$ & $9.35 \pm 0.11$ & 0.019 & 1.9 & $0.63 \pm 0.06$ & $3097 \pm 145$ & $9.26 \pm 0.08$ & 3.1 & 62 \\
\hline NGC 2266 & $0.16 \pm 0.01$ & $3194 \pm 90$ & $8.95 \pm 0.06$ & 0.008 & 2.2 & $0.10 \pm 0.01$ & $3490 \pm 180$ & $8.87 \pm 0.04$ & 3.9 & 41 \\
\hline Berkeley 32 & $0.14 \pm 0.02$ & $3364 \pm 79$ & $9.65 \pm 0.14$ & 0.008 & 3.1 & $0.15 \pm 0.03$ & $3491 \pm 401$ & $9.54 \pm 0.08$ & 4.7 & 40 \\
\hline \multirow[t]{3}{*}{ NGC 2682} & $0.02 \pm 0.02$ & $831 \pm 25$ & $9.50 \pm 0.06$ & 0.019 & 2.8 & $0.05 \pm 0.02$ & $820 \pm 47$ & $9.61 \pm 0.09$ & 3.8 & 335 \\
\hline & $0.05 \pm 0.02$ & $826 \pm 64$ & $9.55 \pm 0.09$ & 0.019 & 2.6 & $0.05 \pm 0.02$ & $820 \pm 47$ & $9.61 \pm 0.09$ & 4.7 & 31 \\
\hline & $0.06 \pm 0.01$ & $910 \pm 16$ & $9.40 \pm 0.05$ & 0.019 & 3.5 & $0.05 \pm 0.02$ & $820 \pm 47$ & $9.61 \pm 0.09$ & 4.8 & 54 \\
\hline \multirow[t]{2}{*}{ NGC 6791} & $0.07 \pm 0.01$ & $3597 \pm 67$ & $10.10 \pm 0.06$ & 0.03 & 1.5 & $0.17 \pm 0.04$ & $4418 \pm 743$ & $9.89 \pm 0.11$ & 2.9 & 100 \\
\hline & $0.06 \pm 0.01$ & $3467 \pm 117$ & $10.15 \pm 0.05$ & 0.03 & 2.1 & $0.17 \pm 0.04$ & $4418 \pm 743$ & $9.89 \pm 0.11$ & 4.2 & 75 \\
\hline \multirow[t]{2}{*}{ NGC 2506} & $0.05 \pm 0.01$ & $2844 \pm 237$ & $9.20 \pm 0.08$ & 0.008 & 1.2 & $0.06 \pm 0.04$ & $3315 \pm 219$ & $9.22 \pm 0.11$ & 1.5 & 284 \\
\hline & $0.05 \pm 0.01$ & $3031 \pm 86$ & $9.30 \pm 0.05$ & 0.008 & 3.1 & $0.06 \pm 0.04$ & $3315 \pm 219$ & $9.22 \pm 0.11$ & 2.6 & 163 \\
\hline \multirow[t]{2}{*}{ NGC 2355} & $0.24 \pm 0.01$ & $1681 \pm 44$ & $9.10 \pm 0.06$ & 0.008 & 2.6 & $0.14 \pm 0.06$ & $2086 \pm 163$ & $8.92 \pm 0.07$ & 6.3 & 217 \\
\hline & $0.18 \pm 0.01$ & $1780 \pm 55$ & $9.00 \pm 0.07$ & 0.008 & 2.4 & $0.14 \pm 0.06$ & $2086 \pm 163$ & $8.92 \pm 0.07$ & 4.1 & 44 \\
\hline \multirow[t]{2}{*}{ Melotte 105} & $0.54 \pm 0.14$ & $2062 \pm 334$ & $8.45 \pm 0.24$ & 0.019 & 1.4 & $0.48 \pm 0.05$ & $2094 \pm 159$ & $8.35 \pm 0.09$ & 1.4 & 289 \\
\hline & $0.56 \pm 0.05$ & $2196 \pm 209$ & $8.35 \pm 0.08$ & 0.019 & 1.3 & $0.48 \pm 0.05$ & $2094 \pm 159$ & $8.35 \pm 0.09$ & 1.3 & 32 \\
\hline \multirow[t]{2}{*}{$\operatorname{Tr} 01$} & $0.61 \pm 0.12$ & $2598 \pm 360$ & $7.90 \pm 0.34$ & 0.019 & 1.1 & $0.57 \pm 0.04$ & $2356 \pm 511$ & $7.48 \pm 0.08$ & 1.3 & 320 \\
\hline & $0.59 \pm 0.04$ & $2464 \pm 153$ & $6.85 \pm 0.56$ & 0.019 & 2.2 & $0.57 \pm 0.04$ & $2356 \pm 511$ & $7.48 \pm 0.08$ & 2.2 & 86 \\
\hline
\end{tabular}


being a cluster member,

$$
W_{i}=e^{\frac{-\left(V_{i}-\left\langle V_{\mathrm{c}}\right\rangle\right)^{2}}{2 \sigma_{V_{\mathrm{c}}}^{2}}} \times e^{\frac{-\left((B-V)_{i}-\left\langle(B-V)_{\mathrm{c}}\right\rangle\right)^{2}}{2 \sigma_{(B-V)_{\mathrm{c}}}^{2}}},
$$

where $V_{i}$ is the $V$ magnitude of the $i^{\text {th }}$ star, $\left\langle V_{\mathrm{c}}\right\rangle$ the average $V$ magnitude of the cluster stars within the $3 \sigma$ error box, $(B-V)_{i}$ the color index of the $i^{\text {th }}$ star and $\left\langle(B-V)_{\mathrm{c}}\right\rangle$ the average color index of the cluster stars within the $3 \sigma$ error box.

\section{Application to observed clusters}

The observational data was obtained from the WEBDA $\dagger$ catalog for the clusters selected from the standard list of Paunzen \& Netopil (2006). We only selected clusters with $U$-band photometry, so that we could determine the reddening using the color-color diagram of open clusters, as done traditionally. We did not use data sets that contained mixed observations from different authors, but used only those that originated from a single source. For the standard list of clusters, only 13 satisfied these conditions. For some clusters we also obtained multiple data sets that satisfied our criteria and those were also used. From the 13 final clusters, we selected a sample of 10 to which we applied our fitting technique. Figure 1 shows an example of the final fit for NGC 2355 and Table 1 includes the final results for all clusters studied.

\section{Conclusions}

We developed a new method to fit isochrones to open cluster data, limiting ourselves to well-studied objects with tabulated Padova isochrones and keeping metallicities fixed to values determined in the literature. The results using the observed data available in the literature show that the parameters determined through our technique are consistent with results obtained by other authors. The Monte Carlo error estimate obtained by our method also gives us a better understanding of the reliability of the results, given the filtering and fitting criteria together with the $\chi_{\text {red }}^{2}$.

We have shown that our method is reliable and robust and although the results are consistent with literature values for the clusters studied, we believe that there is room for improvement in the accepted parameter values for the set of clusters adopted as standards. The possibility of re-evaluating previous results with more quantifiable means is important, as it removes the subjectivity inherent to most open cluster studies to date.

\section{Acknowledgements}

The authors would like to thank CNPq (grant 302762/2007-8), CAPES (project CAPES-GRICES 040/2008) and FAPEMIG (APQ-00090-08).

\section{References}

Paunzen, E. \& Netopil, M. 2006, MNRAS, 371, 1641 